\title{
Volatile profile of Calycanthus occidentalis achenes and evidence for a diverse range of semiochemicals for vespicochory by pestiferous Vespula pensylvanica
}

\author{
John J. Beck ${ }^{1, *}$, Dylan O. Burge ${ }^{2}$, Steve D. Willms ${ }^{1}$ and Nausheena Baig ${ }^{1}$ \\ ${ }^{1}$ Chemistry Research Unit, Center for Medical, Agricultural and Veterinary Entomology, Agricultural \\ Research Service, U.S. Department of Agriculture, 1700 SW $23^{\text {rd }}$ Drive, Gainesville, FL 32608; \\ ${ }^{2}$ Biodiversity Consulting, 550 Vallombrosa Avenue, P. O. Box 451, Chico, CA, 95927, United States.
}

\begin{abstract}
Vespidae represents a large and diverse family and comprises both social and solitary wasps that occupy a large array of habitats. Though some are considered nuisance insects, many of the species are also known as insect predators and pollinators. An additional role of select vespid wasps, originally elucidated approximately 30 years ago and gaining in the number of identified wasps, is dispersal of plant reproductive organs (vespicochory). This relationship is considered mutualistic given the long-range dispersal benefit to the diaspore, and the nutritional benefit to the wasps. Long-chain hydrocarbons have been shown to be responsible for attracting wasps to the organs of some diaspores. Recent observations in northern California indicated that yellowjackets (Vespula spp.), particularly western yellowjacket, Vespula pensylvanica, procured and transported diaspores (achenes) from the western spicebush (Calycanthus occidentalis), a riparian shrub endemic to California. Volatile profile analyses of $C$. occidentalis achenes showed emission of a diverse range of volatile classes. Empirical electrophysiological and trapping studies suggest a composite of odors may stimulate wasp attractancy to the achene.
\end{abstract}

KEYWORDS: attractant, diaspore, achene dispersal, semiochemicals, yellowjacket, vespicochory.

*Corresponding author: john.beck@ars.usda.gov

\section{INTRODUCTION}

Yellowjackets of the genus Vespula are a widespread group of eusocial wasps found throughout the northern hemisphere. The western yellowjacket, Vespula pensylvanica (de Saussure) is a groundnesting pestiferous social wasp native to the western U.S. [1]. In 1979 V. pensylvanica was documented in Hawaii and Maui [2], and it was quickly realized that this invasive species required control measures $[3,4]$ to alleviate pestiferous behavior toward humans, as well as ecological damage. In contrast to these invasive and bothersome characteristics, $V$. pensylvanica provides ecological benefits such as arthropod predation [5] and floral pollination [4].

A more recently identified ecological role of $V$. pensylvanica behavior has been vespicochory the ability to transport and hence disperse plant reproductive organs. Reproductive organs dispersed in this way have an elaiosome containing proteins, fat, carbohydrates, and simple sugars [6,7]. Plant reproductive organ dispersal is performed by a number of abiotic (e.g., wind, water) and biotic (e.g., ants, birds, rodents) means; however, dispersal by vespids is a relatively newly discovered phenomenon, with vespicochory reported for Vespula vulgaris [8, 9], Vespula maculifrons [10], Vespa affinis [11], and Vespa velutina [12-14]. Vespula pensylvanica were recently observed dispersing achenes of the California- endemic shrub Calycanthus occidentalis in northern California [7]. Table 1 summarizes documented observed instances of plant reproductive organ dispersal by Vespidae. 
Table 1. Reported cases of vespicochory, including species of plants and wasps involved.

\begin{tabular}{|l|l|l|c|}
\hline Vespidae (genus sp.) & Plant & State/Country & Ref. \\
\hline Vespula vulgaris & Vancouveria hexandra & Washington, USA & {$[8]$} \\
\hline Vespula vulgaris & Trillium ovatum & Oregon, USA & {$[9]$} \\
\hline Vespula maculifrons & $\begin{array}{l}\text { Trillium cuneatum } \\
\text { T. undulatum } \\
\text { T. catesbaei }\end{array}$ & $\begin{array}{l}\text { North Carolina and South } \\
\text { Carolina, USA }\end{array}$ & {$[10]$} \\
\hline Vespa affinis & Aquilaria malaccensis & India (north east) & {$[11]$} \\
\hline Vespa velutina & $\begin{array}{l}\text { Aquilaria sinensis } \\
\text { Stemona tuberosa }\end{array}$ & China & {$[12]$} \\
China & {$[14]$} \\
\hline Vespula pensylvanica & Calycanthus occidentalis & California, USA & {$[7]$} \\
\hline
\end{tabular}

One possible mechanism for vespid attraction to these plant organs has recently been described in an elegant study by Chen and co-workers [14]. In their work, they describe a mixture of long-chain hydrocarbons from Stemona tuberosa elaiosomes as the odors responsible for attracting Vespa velutina. Other semiochemicals of vespids are well documented and include commercially available traps (e.g., Rescue! $!^{\circledR}$ ) containing heptyl butyrate $[15,16]$, kairomones from insect-produced honeydew [17], fungal-produced volatiles [18], odors from cooked meats [19], and combining heptyl butyrate with chicken extract [20], among others. Longchain cuticular hydrocarbons similar to those extracted from elaiosomes [14] have also been extracted from the bodies of species of yellowjackets, including $V$. pensylvanica [21].

Calycanthus occidentalis Hook. and Arn. (Laurales: Calycanthaceae), commonly known as the spicebush, is a shrub found in the foothills of the Coast, Cascade, and Sierra Nevada mountain ranges in northern California [22]. Spicebush is dependent upon insects for pollination [22], yet no literature exists regarding dispersal of its reproductive organs (achenes). The leaves and twigs of spicebush have been steam distilled and the chemical composition reported [23] to contain primarily cineol, linalyl acetate, borneol, and pinene, among trace amounts of camphor, methyl salicylate, and other sesquiterpene alcohols. No literature currently exists for C. occidentalis achene odors. The objectives of this research were to 1 ) obtain the volatile profile of $C$. occidentalis achenes, 2) survey germane classes of compounds for their ability to elicit electrophysiological responses from $V$. pensylvanica antennae, and 3) probe wasp attractancy via field trapping studies of select chemical classes derived from the seed achene profile and electrophysiological studies.

\section{MATERIALS AND METHODS}

\section{Achene collections}

Achenes used for volatile collections were from the same batches as those collected for wasp observational and behavioural trials as described in Burge and Beck [7], with vouchers deposited at the University California, Davis Center for Plant Diversity. Collected achenes were transported overnight from California to the USDA-ARS Florida laboratory in sealed brown paper bags or sealed 4 or 11-mL scintillation vials with modified lids as described in Beck et al. [24].

\section{Wasp collections}

Individual $V$. pensylvanica were captured at locations in northern California [7] and placed in small plastic containers with modified lids for air exchange. In each container a cotton ball soaked in sugar water was used to maintain the wasps during shipment. The containers were placed in insulated boxes and shipped overnight to the USDA-ARS Florida laboratory for electrophysiological studies. Voucher specimens (D.O. Burge 1024) were deposited at the University of California, Davis, Bohart Museum of Entomology, as well as with the Florida Department of Agriculture and Consumer Services, Division of Plant Industry, Gainesville, Florida.

\section{Achene volatile collection and analysis}

Calycanthus occidentalis achenes (ca. 100) were placed in Wheaton 11-mL vials with a modified cap containing a septum and Teflon liner, similar 
to published methods [24]. The vials were capped and placed in an oven at $40{ }^{\circ} \mathrm{C}$ to mimic warm weather conditions and facilitate full volatile emission from the achenes. Volatiles were allowed to collect (permeate) in the closed system for $60 \mathrm{~min}$ at $40{ }^{\circ} \mathrm{C}$ before insertion of a solid-phase microextraction (SPME, PDMS-DVB, Supelco, Bellefonte, PA, USA) fiber, which was exposed to the headspace volatiles for $30 \mathrm{~min}$ at $40{ }^{\circ} \mathrm{C}$, removed, and immediately thermally desorbed onto an Agilent 7890A gas chromatograph (GC) coupled to a 5975C MSD detector in electron ionization mode (Palo, Alto, CA, USA) outfitted with a J\&W Scientific (Folsom, CA, USA) DB-Wax column $(60 \mathrm{~m} \times 320 \mu \mathrm{m} \times 0.25 \mu \mathrm{m})$ [25]. Each achene collection from the three sites (Table 2) was analysed in triplicate, using a separate set of 100 achenes. For identification and authentication purposes, SPME volatile collections were additionally analysed on an Agilent 7890B GC coupled to a 5977B MSD detector in electron ionization mode, and outfitted with a J\&W Scientific DB-1 column (60 m x $320 \mu \mathrm{m} \times 0.25 \mu \mathrm{m})$. Retention index (RI) values were calculated using a homologous series of $n$-alkanes on both the DB-1 and the DB-Wax columns. RI values from both columns were used to assist with initial identification, and identities were further confirmed by comparison to retention times and fragmentation patterns of standards. Compound identities not verified on both instruments with a commercial or other available standard were marked as tentatively identified, and not included in Table 2. For volatile profile and bioassay result comparison, C. occidentalis achenes were also chemically extracted using the method identical to Burge and Beck [7]. Volatile profiles for the SPME and chemical extraction methods were nearly identical, aside from the expected heavy alkanes extracted from the chemical extraction. Peaks identified as background from the containers, fibers, and columns detected in blanks were removed before analysis.

\section{Electrophysiological studies}

Using methods similar to previously published protocols for honeybees [25], both $V$. pensylvanica antennae were excised and placed on the fork holder using electrode gel. Bioassay discs were loaded with $50 \mu \mathrm{g}$ of each test component using $10 \mu \mathrm{L}$ of solutions of $5 \mu \mathrm{g} / \mu \mathrm{L}$ in pentane. After solution addition, the pentane was allowed to evaporate for $1 \mathrm{~min}$ and the discs placed in a Pasteur pipette, which was then inserted into a volatile tube leading to the insect antennae. All electrophysiological studies were performed using a 4-channel acquisition controller, electrode fork holder, and pre-amplifier (Syntech, Kirchzarten, Germany). A 0.5 s pulse flow $(300 \mathrm{~mL} / \mathrm{min})$ and a humidified continuous flow $(125 \mathrm{~mL} / \mathrm{min})$ directed odors through an air and volatile tube $(1.5 \mathrm{~cm}$ diameter) containing the mounted antennae and probe. A Faraday cage was used to protect against ambient electrical interference. To account for variability in response among individuals, responses to blanks (10 $\mu \mathrm{L}$ pentane loaded onto a bioassay disc) were subtracted from each sample and antennal response values then normalized to the standard stimulus (50 $\mu \mathrm{g}$ of benzaldehyde) set at $100 \%$ (Table 3 ).

\section{Field trapping studies}

Wasp capture used identical techniques as previously published [7] using Rescue! ${ }^{\circledR}$ traps, but instead of the achene extracts loaded onto bioassay discs as in Burge and Beck [7], pure individual compounds or blends of pure compounds were loaded into 2.0 $\mathrm{mL}$ conical vials and sealed until the field trials. Prior to placement in the field, the vial caps were replaced with caps containing a $2 \mathrm{~mm}$ hole, and with cotton strips (ca. $1 \times 3 \mathrm{~cm}$ ) inserted. For all trapping studies, each compound was evaluated with no solvents to dilute the compounds or the blends (neat). For individual compounds, $180 \mu \mathrm{L}$ were added into each vial ( $\mathrm{n}=5$ or 6 ) for each lure (Table 4). For blends, the total volume added was $180 \mu \mathrm{L}$; thus, a three-component mixture comprised $60 \mu \mathrm{L}$ of each compound. Traps baited with heptyl butyrate were used as a positive control to confirm wasp population at each site [7]. Field trapping trials were performed at the University of California La Kretz Center Field Station, located in the western Santa Monica Mountains (Trial 1; Table 4; 34.0970, -118.8156) and at Elysian Park, located in the eastern Santa Monica Mountains (Trial 2; Table 4; 34.0841, -118.2462).

\section{RESULTS AND DISCUSSION}

The volatile profiles of the achenes were surprisingly high in the number of compounds and chemical class diversity. A total of 102 compounds were detected consistently in the three batches of achenes analysed by SPME GC-MS. Table 2 provides the 44 authenticated compounds reported by relative abundances, and illustrates the wide diversity 


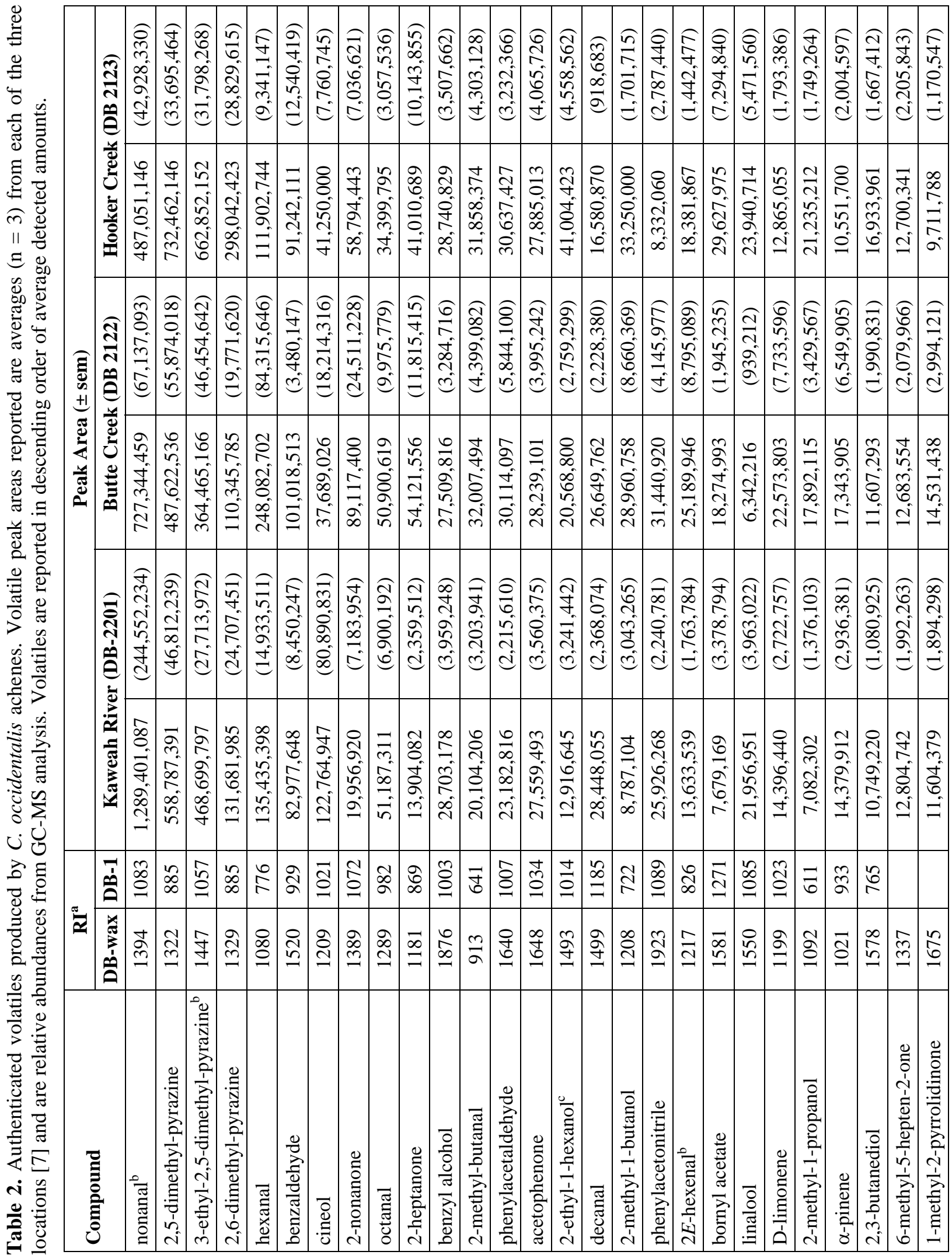




\begin{tabular}{|c|c|c|c|c|c|c|c|c|c|c|c|c|c|c|c|c|}
\hline$\frac{6}{6}$ & 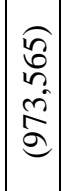 & $\begin{array}{l}\hat{\sigma} \\
\vec{b} \\
-1 \\
\hat{I} \\
\hat{\theta}\end{array}$ & 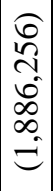 & 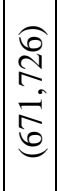 & 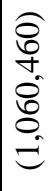 & 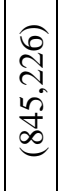 & 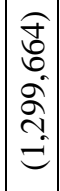 & 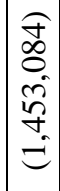 & 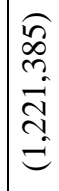 & 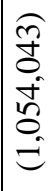 & $\mid \begin{array}{l}\widetilde{\widehat{D}} \\
\tilde{\Omega} \\
\tilde{\omega} \\
\tilde{\omega}\end{array}$ & $\mid \begin{array}{l}0 \\
b \\
L \\
\tilde{N} \\
\hat{D} \\
\mid\end{array}$ & 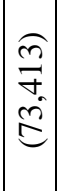 & 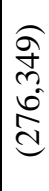 & 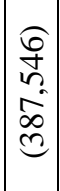 & 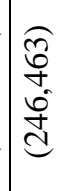 \\
\hline J & 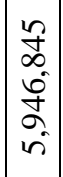 & $\mid \begin{array}{c}\overrightarrow{\tilde{n}} \\
0 \\
0 \\
0 \\
0 \\
\overrightarrow{-}\end{array}$ & 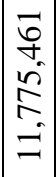 & 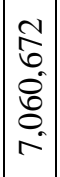 & 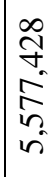 & 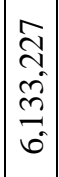 & 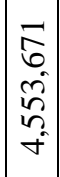 & 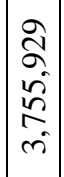 & 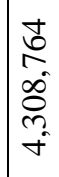 & 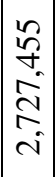 & 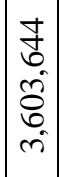 & 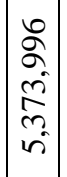 & $\begin{array}{l}\infty \\
\infty \\
\infty \\
\hat{\omega} \\
\tilde{\alpha} \\
\hat{v}\end{array}$ & $\begin{array}{l}\text { J } \\
\infty \\
\infty \\
\tilde{D} \\
\infty \\
i \\
i\end{array}$ & 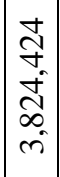 & 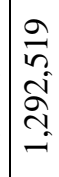 \\
\hline $\begin{array}{l}\dot{b} \\
\stackrel{\sim}{\sim}\end{array}$ & 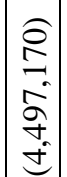 & 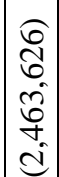 & \begin{tabular}{|l}
$\hat{y}$ \\
$\alpha$ \\
$\tilde{y}$
\end{tabular} & $\left|\begin{array}{c}f \\
0 \\
\infty \\
\vec{G} \\
0\end{array}\right|$ & $\mid \begin{array}{l}\sigma \\
\text { g } \\
\text { i } \\
\hat{\delta}\end{array}$ & $\mid \begin{array}{c}\widehat{\widetilde{N}} \\
\underline{0} \\
0 \\
\tilde{0}\end{array}$ & $\mid \begin{array}{c}\widehat{\sigma} \\
\dot{q} \\
o \\
o \\
\vec{t} \\
\mathfrak{d}\end{array}$ & $\begin{array}{l}\widehat{\overrightarrow{\tilde{D}}} \\
\hat{\sim} \\
\tilde{\tilde{D}} \\
\tilde{d}\end{array}$ & 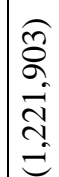 & సิ & 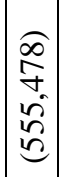 & 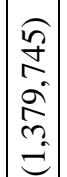 & 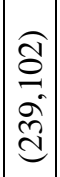 & $\begin{array}{l}0 \\
6\end{array}$ & $\mid \begin{array}{l}0 \\
0 \\
0 \\
0 \\
0 \\
0 \\
0 \\
0\end{array}$ & 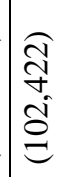 \\
\hline
\end{tabular}

\&

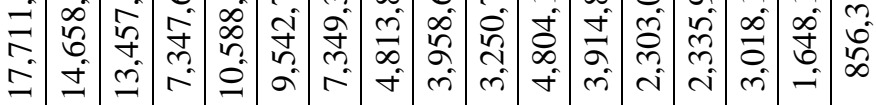

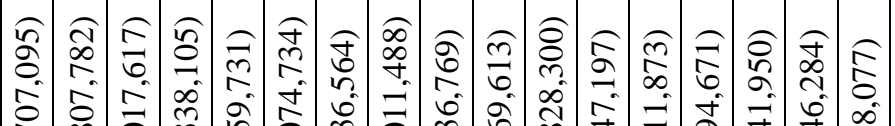

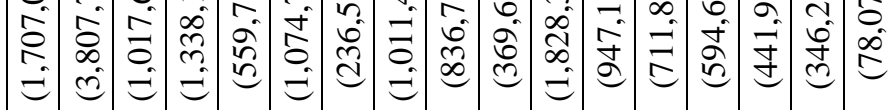

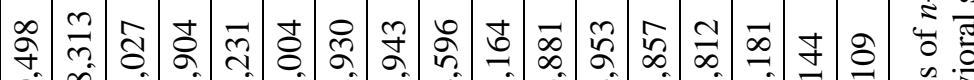

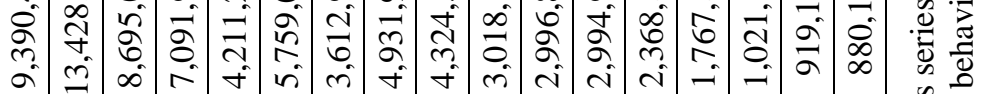

\begin{tabular}{|c|c|c|c|c|c|}
\hline 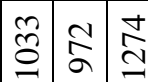 & 岕 & 员 & : & $\begin{array}{l}\mathscr{0} \\
\sigma\end{array}$ & 妾 \\
\hline
\end{tabular}

恿

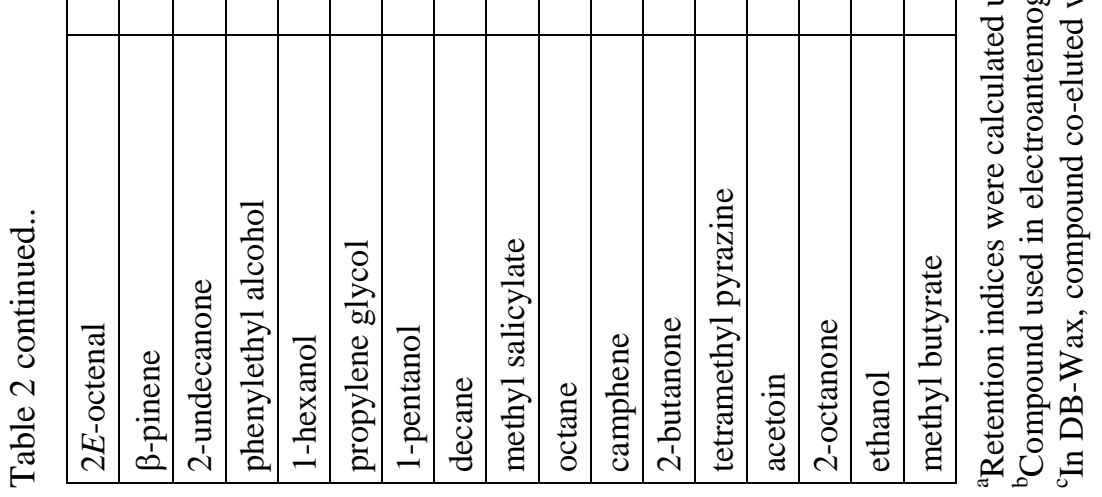


Table 3. Electrophysiological studies of representative classes of detected compounds from $C$. occidentalis achenes or purported semiochemicals, and elicited responses from $V$. pensylvanica antennae.

\begin{tabular}{|c|c|c|}
\hline Compound class & Compound & $\begin{array}{c}\text { Antennal response } \\
\text { (\%, } \boldsymbol{n}=\mathbf{6 - 8} \text { wasps) }^{\mathbf{a}}\end{array}$ \\
\hline \multirow{2}{*}{ long-chain alkane } & tetracosane & $22.6 \pm 9.1$ \\
\cline { 2 - 3 } & nonacosane & $24.2 \pm 11.8$ \\
\hline \multirow{2}{*}{ aldehyde } & (2E)-hexenal $^{\mathrm{b}}$ & $290.3 \pm 63.2$ \\
\hline & nonanal $^{\mathrm{b}}$ & $146.9 \pm 55.8$ \\
\hline acid & octanoic acid $^{\mathrm{b}}$ & $150.0 \pm 65.7$ \\
\hline alcohol & 1-octanol $^{\mathrm{b}}$ & $273.0 \pm 65.2$ \\
\hline aromatic & 2-ethyl-3,5-dimethylpyrazine $^{\mathrm{b}}$ & $613.8 \pm 537.1$ \\
\hline
\end{tabular}

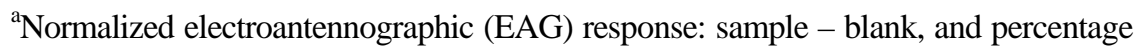
of positive control, benzaldehyde, average \pm sem

${ }^{\mathrm{b}}$ Either that compound or the class of compounds was detected

Table 4. Field trapping studies of $V$. pensylvanica for select classes detected from C. occidentalis achenes, or purported semiochemicals ( $n=5$, trial $1 ; n=6$, trial 2).

\begin{tabular}{|l|c|c|c|}
\hline Compound or blend & Trial 1 & Trial 2 & Combined \\
\hline tetracosane (24) & $1.0 \pm 0.5$ & $6.8 \pm 3.2$ & $4.2 \pm 1.9$ \\
\hline nonanal (9AL) & $1.6 \pm 0.5$ & $9.5 \pm 7.1$ & $5.9 \pm 3.9$ \\
\hline 1-octanol (8OH) & $5.2 \pm 1.7$ & $16.8 \pm 9.4$ & $11.6 \pm 5.3$ \\
\hline 2-ethyl-3,5-dimethylpyrazine (EDMP) & $0.0 \pm 0.0$ & $0.0 \pm 0.0$ & $0.0 \pm 0.0$ \\
\hline blend 24/9AL/8OH/EDMP & - & $0.3 \pm 0.2$ & $0.3 \pm 0.2$ \\
\hline blend 24/9AL/8OH & - & $1.2 \pm 0.2$ & $1.2 \pm 0.5$ \\
\hline
\end{tabular}

of chemical classes detected. Generally classified but not fully authenticated, the total profile comprised: 17 aromatics (e.g., furans, pyrazines); 16 aldehydes; 13 ketones; 11 alkenes; 7 benzenoids; 7 monoterpenes; 7 primary alcohols; 5 various alkanes; 2 each of sesquiterpenes and unknown compounds; and, 1 each of other classes.

Given the unexpectedly large number of detected compounds and range of classes, it is surprising that the western yellowjacket ( $V$. pensylvanica) is known to be attracted to a single compound, namely heptyl butyrate (a simple ester) [15, 16]. This is nicely demonstrated by the success of the commercial attractant Rescue! ${ }^{\circledR}$ which contains only heptyl butyrate. However, when presented with a complex bouquet of volatiles emitted from a food source, there is likely more than one compound or class of compounds that provides the attractiveness of the food source. Indeed, $V$. pensylvanica were shown to be attracted to the primary alcohols 2-methyl-1-butanol, 3-methyl-1butanol, and 2-phenylethanol, volatiles produced by fungal colonies of Aureobasidium pullulans [18]. Moreover, a relatively complex mixture of volatiles from the honeydew of insects was shown to attract $V$. vulgaris [17]. The authors of this study [17] also demonstrated wasp attraction to simpler blends including aromatics, an aldehyde, a secondary alcohol, and a primary alcohol. Conversely, Zhang and co-workers [26] showed repellency of $V$. pensylvanica using essential oils that included compound classes of terpenoids, aromatics, and a secondary alcohol. Interestingly, in their 2013 repellency study [26] methyl salicylate and 3octanol were noted as providing significant repellency of $V$. pensylvanica, whereas Brown's 2015 study [17] on $V$. vulgaris noted these two compounds as 
slight attractants when presented individually during trapping studies. Semiochemical activity (either attractant or repellent) of the primary alcohol 1octanol was corroborated in the present study (Table 3) and also attracted the most $V$. pensylvanica in our trapping studies (Table 4).

This dichotomy of class and compound semiochemical behavior was also indirectly noted in the pyrazine class of compounds, which as reported in Table 2 were amongst the most highly emitted compounds from $C$. occidentalis achenes. Literature regarding $V$. pensylvanica attraction to cooked meat is abundant, and cooked meat is known to be a source of proteins [3, 19, 20]. Interestingly, it is also known in the literature that pyrazines (and aldehydes) are commonly found in the headspace of cooked meats [27, 28]. In the present study, the compound 2-ethyl-3,5dimethylpyrazine elicited the highest response from $V$. pensylvanica antennae (Table 3), yet when tested individually during wasp trapping studies (Table 4) this compound did not attract any $V$. pensylvanica. When included in a blend of volatiles with other tested compounds, the results suggested that 2-ethyl-3,5-dimethylpyrazine may inhibit attraction of $V$. pensylvanica.

While little is known about the role of pheromones in vespids [29], pyrazines have been reported as sex pheromones of Eurytomidae [30] and Thynnidae wasps [31]. However, long-chain hydrocarbons have been detected on the cuticles of several species of yellowjackets, including $V$. pensylvanica [21], and long-chain hydrocarbons have also been shown to signal fertility in some vespids [32]. Interestingly, several long-chain hydrocarbons were detected in the elaiosomes of Stemona tuberosa, a seed dispersed by Vespa velutina [14]. Further work by the same authors showed that these same longchain hydrocarbons were the volatiles involved in the attraction of Vespa [14]. In the present study we confirmed both the electrophysiological activity (Table 3) and attractancy (Table 4) of the long-chain hydrocarbon tetracosane, a component detected in the Chen et al. [14], the Derstine et al. [21], and van Zweden et al. [32] studies.

\section{CONCLUSION}

The broad range of volatile compound classes detected from the achenes of Calycanthus occidentalis, and the noted electrophysiological and behavioral responses provide evidence for a potential complex blend of semiochemicals for $C$. occidentalis vespicochory. This hypothesis is supported by the association of some of these food-related compounds detected in the present study to known attractants of $V$. pensylvanica. Some examples include: 1) pyrazines, which are indicative of a source of protein, albeit the trapping study results for the chosen pyrazine suggested an inhibitory activity, 2) the aldehydes hexanal, nonanal, and octanal, which are indicative of lipid oxidation [28], and 3) the primary alcohols phenylethanol and 2-methyl1-butanol, which have been detected from a fungus that has been reported as a source of food for wasps [18]. Further investigations into the volatile profiles of the plant reproductive organs from the first four studies [8-11] listed in Table 1, along with in-depth electrophysiological and behavioral studies may reveal definitively the identities of the semiochemicals responsible for their noted vespicochory.

\section{ACKNOWLEDGEMENTS}

Research was conducted under USDA-ARS Research Project 6036-22000-028-00D.

\section{CONFLICT OF INTEREST STATEMENT}

The authors declare no conflicts of interest.

\section{REFERENCES}

1. CABI. 2018, Vespula pensylvanica. In: Invasive Species Compendium, Wallingford, UK: CAB International.

2. Nakahara, L. M. 1980, Hawaii Dept. of Agriculture, Honolulu, Hawaii.

3. Hanna, C., Foote, D. and Kremen, C. 2012, Pest Manag. Sci., 68, 1026-1033.

4. Hanna, C., Foote, D. and Kremen, C. 2013, J. Appl. Ecol., 50, 147-155.

5. Gambino, P. 1992, Proc. Hawaiian Entomol. Soc., 31, 157-164.

6. Lisci, M., Bianchini, M. and Pacini, E. 1996, Flora, 191, 131-141.

7. Burge, D. O. and Beck, J. J. 2019, Madroño., Accepted, in print.

8. Pellmyr, O. 1985, Madroño, 32, 56.

9. Jules, E. S. 1996, Am. Midl. Nat., 135, 367-369. 
10. Zettler J. A., Spira T. P. and Allen C. A. 2001, Am. Midl. Nat., 146, 444-446.

11. Manohara, T. N. 2013, Curr. Sci., 105, 298-299.

12. Chen, G., Liu, C. and Sun, W. 2016, Plant Div., 38, 227-232.

13. Chen, G., Wang, Z. -W., Qin, Y. and Sun, W. -B. 2017, J. Integ. Plant Biol., 59, 792-796.

14. Chen G., Wang Z. -W., Wen P., Wei W., Chen, Y., Ai, H. and Sun W. -B. 2018, New Phytol., 220, 714-725.

15. Davis, H. G., Eddy, G. W., McGovern, T. P. and Beroza, M. 1969, J. Econ. Entomol., 62, 1245.

16. Landolt, P. J., Reed, H. C. and Ellis, D. J. 2003, Fla. Entomol., 86, 323-328.

17. Brown, R. L., El-Sayed, A. M., Unelius, C. R., Beggs, J. R. and Suckling, D. M. 2015, J. Chem. Ecol., 41, 1018-1027.

18. Davis, T. S., Boundy-Mills, K. and Landolt, P. J. 2012, Microb. Ecol., 64, 1056-1063.

19. Grant, C. D., Rogers, C. J. and Lauret, T. H. 1968, J. Econ. Entomol., 61, 1653-1656.

20. Liang, D. and Pietri, J. E. 2017, Insects, 8, 17.

21. Derstine N. T., Gries R., Zhai H., Jimenez S. I. and Gries G. 2018, Insectes Sociaux, 65, 581-591.

22. Grant, V. 1950, Am. J. Bot., 37, 294-297.

23. Scalione C. C. 1916, J. Ind. Eng. Chem., 8, 729-731.
24. Beck, J. J., Baig, N., Cook, D., Mahoney, N. E., and Marsico, T. D. 2014, J. Agric. Food Chem., 62, 12273-12276.

25. Rering, C. C., Beck, J. J., Hall, G. W., McCartney, M. M. and Vannette, R. L. 2018, New Phytol., 220, 750-759.

26. Zhang, Q. -H., Schneidmiller, R. G. and Hoover, D. R. 2013, Pest Manag. Sci., 69, 542-552.

27. Mussinan, C. J., Wilson, R. A. and Katz, I. 1973, J. Agric. Food Chem., 21, 871-872.

28. Herrara-Jiménez, M., Escalona-Buendía, H., Ponce-Alquicira, E., Verde-Calvo, R. and Guerrero-Legarreta, I. 2007, Int. J. Food Prop., 10, 807-818.

29. Derstine, N. T., Ohler, B., Jimenez, S. I., Landolt, P. and Gries, G. 2017, Entomol. Exp. Appl., 164, 35-44.

30. Mori, K. and Yang, C. Y. 2017, Tetrahedron, 73, 4766-4769.

31. Bohman, B., Phillips, R. D., Menz, M. H. M., Berntsson, B. W., Flematti, G. R., Barrow, R. A., Kixon, K. W. and Peakall, R. 2014, New Phytol., 203, 939-952.

32. van Zweden, J. S., Bonckaert, W., Wenseleers, T. and d'Ettorre, P. 2014, Evolution, 68, 976-986. 